Review

\title{
NASA's Exploration Missions to the Red Planet
}

\author{
Santosh Kosambe \\ Independent Researcher, Pune, India
}

Article history

Received: 24-05-2019

Revised: 11-06-2019

Accepted: 05-07-2019

Email: santo2040@gmail.com

\begin{abstract}
The exploration missions to the red planet were started in 1960 by the Soviet Union, since then the planet has gain attention of all the scientists and explorers worldwide. Several exploration missions have been launched by the space organizations and nations to explore the Martian surface. NASA launched its first exploration mission to Mars in 1964 in the form of Mariner 3. After that, the series of robotic exploration missions have been launched to understand the red planet intensely. The Mars Exploration Program (MEP) was also formed in 1993 to explore the possibilities of the presence of life, climate and natural resources on Mars. The MEP uses the spacecraft, orbiters, landers and rovers to explore the Martian soil. As of present, NASA has launched twenty-five missions to the red planet out of which only five missions were unsuccessful. Some of the significant discoveries have been made in recent years with missions such as Pathfinder, Spirit, Opportunity, Curiosity, MAVEN and InSight. Currently, NASA is making plans to send more robotic explorers on the Martian soil in the upcoming years to make more discoveries and gain scientific information. These robotic missions are the first steps towards the human-crewed missions to Mars. The present paper provides a quick overview of NASA's past, present and future robotic exploration missions to the red planet.
\end{abstract}

Keywords: NASA, Red Planet, Mars Exploration, Martian, Rover, Lander

\section{Introduction}

Mars also known as Red Planet, is a unique dusty, cold, dry desert world which has been a prime target for all space-faring nations and agencies throughout the world. As the fourth planet in our solar system, it has captured the attention of scientists and researcher's over the years due to its resemblance to our own planet. Also, it is the only planet in our solar system where the signs of water and life have been traced. Several exploration missions have been launched to the red planet due to the presence of a weather, seasons, dust storms, ice caps in the polar region, colossal volcanoes, canyons and a thin atmosphere made up of $95 \%$ of Carbon Dioxide, $2 \%$ of Nitrogen and $1 \%$ of Argon gas. Previous NASA missions have found the evidence of Mars being a much wetter and warmer planet with a thicker atmosphere. No planet in our solar system beyond Earth has been studied as intensely as Mars. Mars has situated 1.5 Astronomical Unit (AU) from the Sun due to which it takes 13 minutes for light to travel from Sun to Mars. Mars has two moons named Phobos and Deimons. Since 1994, NASA studies Mars as one of the planetary systems under the Mars Exploration Program (MEP). The MEP is formed to understand the process of formation of the planet, evolution history of the planet, the past geological activities that help to shape the planet across time, the possibility of the planet to sustain life and the future human exploration of the planet. The MEP, formerly known as Mars Surveyor Program (MSP) will help us to recognize the potentiality of the red planet to sustain life using spacecraft, orbiters, rovers and landers. Also, it helps us to better understand the questions like whether there was, there is, or there will be a life on Mars. The answers to all these questions will be obtained by understanding the climate of the planet, geological activities, its atmosphere and environment and several other processes that have interacted to shape the Mars over time. The current exploration missions at NASA are following a science theme under the MEP known as 'Seek Signs of Life' to seek the possibilities of past, present and future life on the red planet. As water is a key to the life on any planet, past NASA missions such as Mars Odyssey, Mars Exploration Rovers, Mars Reconnaissance Orbiter and Mars Phoenix Lander were launched to find a clue of liquid water on Mar's Surface under the MEP science theme 'Follow the water.' NASA's MEP follows four broad goals to seek the potentiality of the planet to sustain life in the past, present, or future. 
Table 1: Comparison of specifications of Mars and Earth

\begin{tabular}{lll}
\hline & Mars & Earth \\
\hline Equatorial Radius & $3,396.2 \mathrm{~km}$ & $6,378.1 \mathrm{~km}$ \\
Gravity & $3.711 \mathrm{~m} / \mathrm{s}^{2}$ & $9.807 \mathrm{~m} / \mathrm{s}^{2}$ \\
Natural Satellites & 2 & 1 \\
Mean Density & $3,933 \mathrm{~kg} / \mathrm{m}^{3}$ & $5,514 \mathrm{~kg} / \mathrm{m}^{3}$ \\
Tilt of Axis & $25.2^{0}$ & $23.43^{0}$ \\
Orbit Eccentricity & 0.0933941 & 0.01671123 \\
Duration of Day & 24.623 hours & 23.934 hours \\
Duration of Year & 687 days & 365 days \\
Surface Temperature & $-63^{\circ} \mathrm{C}$ & $14^{\circ} \mathrm{C}$ \\
Pressure & 7.5 millibars & 1,013 millibars \\
Distance from Sun & $227,943,824 \mathrm{~km}$ & $149,598,262 \mathrm{~km}$ \\
Polar Caps & Covered with a mixture & Permanently covered \\
& of Carbon Dioxide ice & with water ice \\
Composition of & and water ice & Nitrogen $(78.09 \%)$, \\
Atmosphere & Carbon Dioxide $(96 \%)$, & Oxygen $(20.95 \%)$, \\
& Nitrogen $(1.89 \%)$, Argon $(1.93 \%)$, & Argon $(0.93 \%)$, \\
Largest Volcanoes & Oxygen $(0.145 \%)$, Water vapor & Carbon Dioxide $(0.039 \%)$ \\
& $(0.03 \%)$, Nitric Oxide $(0.01 \%)$ & Manua Loa \\
Deepest Canyon & Olympus Mons & $10.13 \mathrm{~km}$ high \\
& $26 \mathrm{~km}$ high & $121 \mathrm{~km}$ in diameter \\
\hline
\end{tabular}

The first goal is designed to understand whether a planet having a high potential for habitability and for preserving biosignatures contains any evidence of past or extant life. The second goal is designed to understand the processes interacted in the evolution of climate on the red planet. Also, to understand the several processes and the present and past history of climate on the red planet under current and different orbital configurations. The third goal is designed to determine the beginning and evolution of Mars geology by studying the composition, structure, dynamics and it's interior. The fourth and last goal is designed to acquire complete information of the red planet with the robotic exploration missions which will eventually help us to prepare manned missions to red planets orbit, to the Mars surface, to the surface of Mars moons (Phobos or Deimos) with minimum risk, cost and performance (NASA, 2019a). The comparison of specifications of Mars and Earth is shown in Table 1.

\section{Past Missions to Mars}

In between 1962 to late 1973, NASA's Jet Propulsion Laboratory (JPL) designed and built ten spacecraft's named Mariner to explore the planets like Venus, Mars and Mercury in the inner solar system for the first time. All these spacecraft were small orbiters weighing less than 500 $\mathrm{kg}$. Each spacecraft was designed with solar panels and a dish antenna which would be pointed towards the Sun and the Earth respectively. Also, several scientific instruments were placed on these spacecraft's depending upon the object they were studying. The unique feature of the Mariner was they were designed as three-axis stabilized spacecraft. Each of the Mariner Project consisted of two spacecraft. Unfortunately, out of the ten spacecraft's designed, three spacecraft's namely Mariner 1 and Mariner 8 was utterly destroyed during the launch and Mariner 3 was lost entirely after the launch.

\section{Mariner 3}

Mariner 3 was the first spacecraft in Mariner project which was designed to perform the first flyby mission to Mars. The launch of Mariner 3 took place on November 5, 1964. The launch was successful, but the shroud present at the top of the rocket in which the spacecraft was placed failed to open and the dream of Mariner 3 to become the first spacecraft to perform a flyby to Mars was ended (NASA, 2019b).

\section{Mariner 4}

Mariner 4 became the first spacecraft to perform the flyby to Mars on July 14, 1965. It was the second spacecraft in the Mariner project designed to perform a flyby mission to Mars. The launch of the Mariner 4 took place on November 28, 1964. Mariner 4 was also the first spacecraft to take close-up images of the interplanetary body for the first time. The images taken by the spacecraft showed impact craters on Mars. Mariner 4 was lasted for three years studying the solar wind (Fig. 1) (Reiff, 1966). 


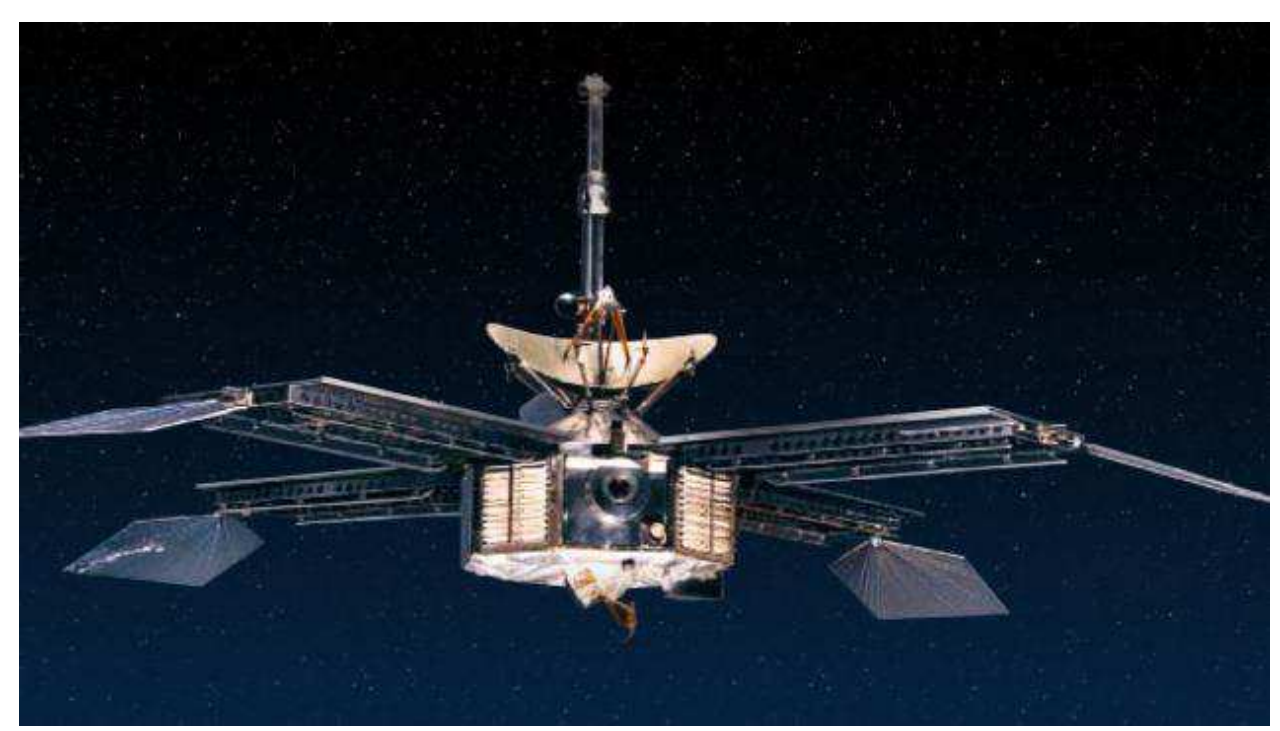

Fig. 1: Mariner 4 Spacecraft

\section{Mariner 6}

Mariner 6 was the second pair in the Mariner project designed to perform the flyby mission to Mars. It was launched on February 24, 1969, on Atlas-Centaur. The Mariner 6 performed the successful flyby over the equator region and south-polar region of Mars on July 31,1969 . The spacecraft analyzed the atmosphere and surface of the red planet using remote sensors and also captured multiple images as well. The Mariner 6 spacecraft is shown in Fig. 2 (NASA, 1969).

\section{Mariner 7}

Mariner 7 was the second spacecraft in the next Mariner project designed to perform the flyby mission to Mars. It was also launched on Atlas-Centaur on March 27, 1969. The Mariner 7 also flew over the equator and south-polar region of the red planet on August 5, 1969. The spacecraft analyzed the atmosphere and surface of the red planet using remote sensors and also captured multiple images as well (NASA, 1969).

\section{Mariner 8}

Mariner 8 was designed to be the first Mars orbiter to perform its operation in orbit around Mars. The launch of Mariner 8 took place on May 8, 1971, but, it failed during its launch (NASA, 1973).

\section{Mariner 9}

The launch of Mariner 9 took place on May 30, 1971, on Atlas-Centaur. On November 14, 1971, it became the first artificial satellite to perform its operation in orbit around Mars. The spacecraft detected the dust storms in Martian atmosphere. Also, it detected the volcanoes and canyons on Mars. Mariner 9 was the first spacecraft to complete $100 \%$ photo-mapping of Martian surface. It also captured the close-up pictures of Mars two small moons, Phobos and Demons. The spacecraft ended its mission on October 27, 1972. Mariner 9 is shown in Fig. 3 (Steinbacher and Haynes, 1973).

\section{Viking Project}

Viking was known to be the NASA's first spacecraft to land a lander on the planet's surface successfully. The project consists of two identical spacecraft's named as Viking-1 and Viking-2. The two identical pairs of lander and orbiter were also designed and built under the Viking Project. Both the spacecraft were launched from Florida atop Titan-III Centaur rocket. The launch of Viking-1 took place on August 20, 1975. It takes nearly a year for Viking-1 to reach Mars and finally on June 19, 1976, it reached Mars orbit. Upon orbit insertion, the lander gets detached and started its journey towards the Martian surface. On July 20, 1976, the Viking-1 lander landed successfully on the western slope of Chryse Planitia at 22.30 latitude and 480 longitude. The launch of Viking-2 took place on September 9, 1975. After completing the journey of nearly 11 months, Viking-2 reached in Mars orbit on August 7, 1976. After the insertion, the lander gets separated and accelerated towards the surface of the red planet. On September 3, 1976, the Viking-2 lander got settled down on Martian soil at Utopia Planitia at 47.70 latitude and 480 longitude. Both the spacecraft consists of two orbiters weighing $2,325 \mathrm{~kg}$ with fuel. The orbiters were 3.3 meters high and 9.7 meters across the extended solar panels. The avionics of the orbiter was mounted on side faces of the structure. The orbiters also consist of two nickel-cadmium batteries to supply power in the absence of sunlight. The orbiters were designed with a lifetime of 
four to six months in orbit. The two landers weighing $576 \mathrm{~kg}$ was approximately 3 meters across and 2 meters tall. Both the orbiter and lander consists of several scientific instruments to perform science experiments and to record data. As soon as the landers touched down on Mars soil, they started collecting scientific data from the Martian surface. The seismometer on Viking-1 failed after its landing and the seismometer on Viking-2 detected only one seismic event. Scientific instruments weighing $91 \mathrm{~kg}$ were mounted on two landers to perform the three biological experiments which were designed to seek any evidence of life on the red planet. These experiments do not find any signs of the presence of microbial life on the planet's surface. Although experiments revealed unpredicted and inexplicable chemical activities in the planet's soil. The lander measured physical properties and composition of the planet's upper atmosphere. Also, landers measured the physical and magnetic properties of soil along with its elemental composition. Numerous high-quality pictures have been captured by both the lander $(4,500)$ and orbiters $(52,000)$. The orbiters mapped nearly $97 \%$ of the planet's surface. Viking also discovered the presence of Nitrogen and water ice on the planet. After significant discoveries, Viking-1 and Viking-2 orbiters concluded their mission on August 17, 1980 and July 25, 1978, respectively. Also, Viking-1 and Viking-2 landers ended their exploration of the red planet on November 23, 1982 and April 11, 1980 (Fig. 4) (Soffen and Snyder, 1976) (Moore et al., 1977).

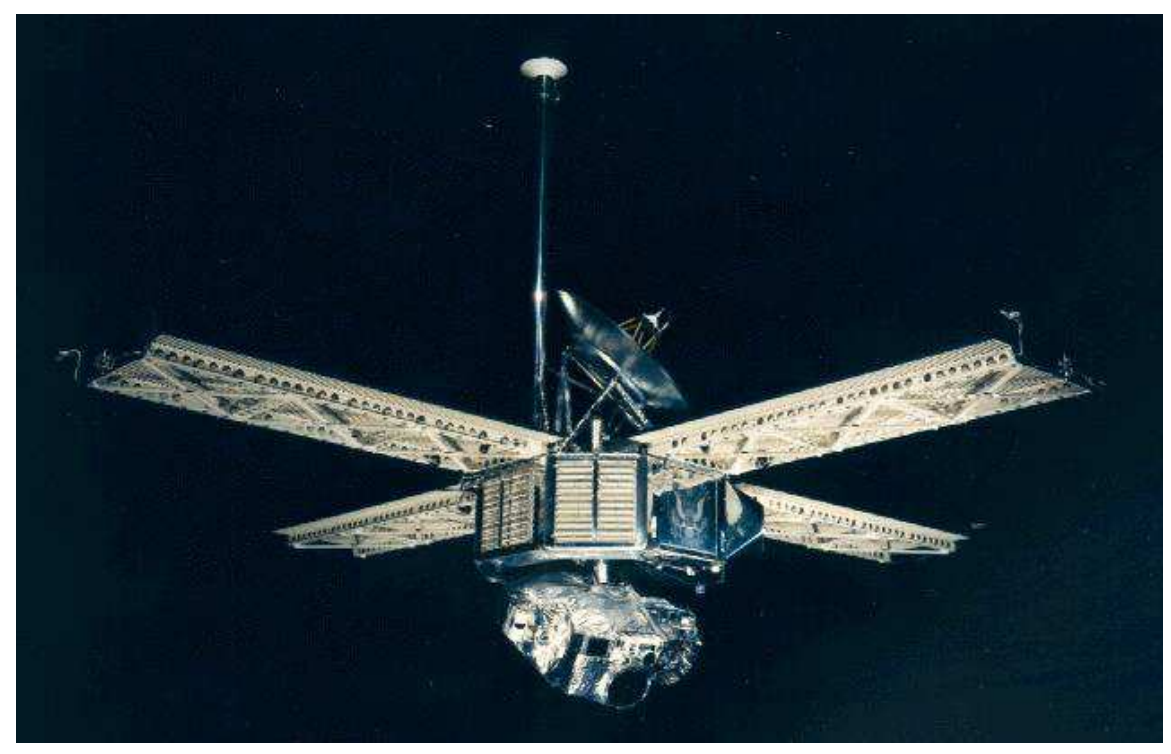

Fig. 2: Mariner 6 Spacecraft

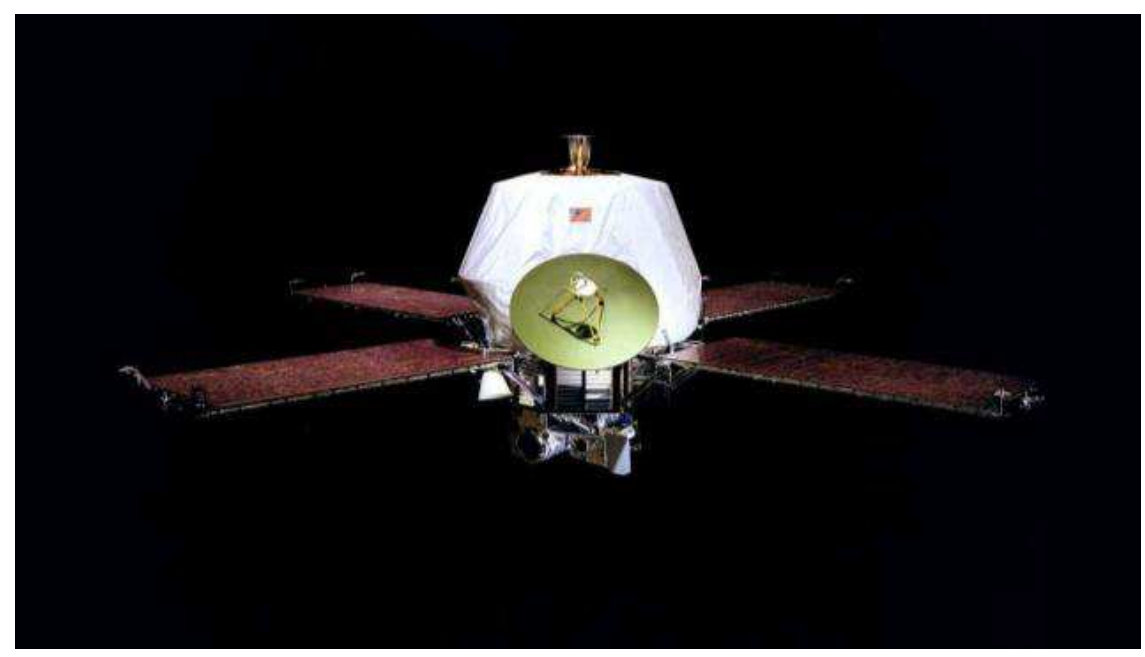

Fig. 3: Mariner 9 Spacecraft 


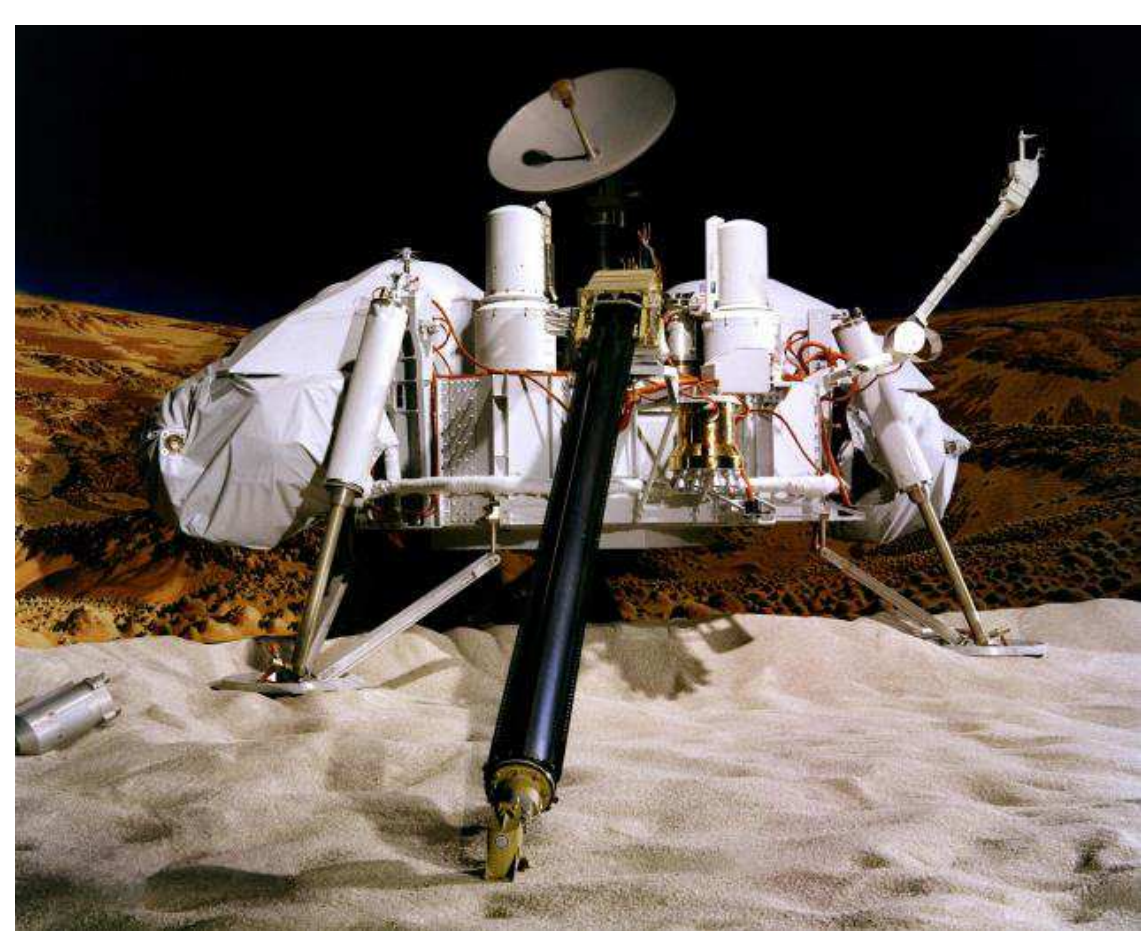

Fig. 4: Viking-1 and Viking-2 Lander

\section{Mars Observer}

Nearly seventeen years after the successful missions to Mars, NASA launched its first spacecraft in the form of Mars Observer to study the geoscience and atmosphere of the red planet. The launch of Mars Observer, also known as Mars Geoscience and Climate Orbiter (MGCO) took place on September 22, 1992, on expandable Titan-III rocket. The Earth-orbiting communication satellite was converted into an orbiter for the mission. The spacecraft consists of eight number of science instruments to carry out a detailed study of the Martian surface. Three days before the scheduled orbit insertion, on August 21, 1993 contact with the spacecraft was lost completely. The mission was ended with disappointment on August 22, 1993 (Fig. 5) (JPL, 1993).

\section{Mars Global Surveyor}

Mars Global Surveyor (MGS) was one of the first in a series of Mars Surveyor Program (MSP) designed to explore the entire red planet. The launch of spacecraft took place on a Delta-II rocket on November 7, 1996. The mission was designed to gather information of the planet's interior along with its surface and atmosphere. Also, the objectives of the mission were to map the entire planet while understanding the climate, geography and resources. The spacecraft entered into the Mars orbit on September 1997. The six science instruments on the spacecraft started to collect the measurement from an altitude of $378 \mathrm{~km}$ for 687 days. The images captured by Mars Orbital Camera on-board the spacecraft shows the presence of gullies and flow of debris along with deposits of dirt and rocks transported by these flows. Also, the reading from magnetometer finds out that the planets magnetic field was confined to specific regions. Close-up pictures and temperature patterns revealed the planets moon Phobos is made up of powdery material due to the impact of meteoroids. Extreme temperature variation is also seen during the bright and dark side of the moon. The Thermal Emission Spectrometer (TES) indicated the evidence of an ancient hydrothermal system on the planet's surface. The laser altimeter helps scientists to plot the first 3D view of northern polar ice caps of Mars. After studying continuously for eight years spacecraft ended its mission on November 14, 2006 (Fig. 6) (Albee et al., 2001).

\section{Mars Pathfinder}

The Mars Pathfinder was first completed robotic mission developed under NASA's Discovery Program. The low-cost mission was designed to demonstrate a new technique to deliver a pair of a lander and first rover on the Martian surface. The launch took place on December 4, 1996, atop a Delta-II 7925 rocket. After completing its seven-month journey, the spacecraft weighing $584 \mathrm{~kg}$ entered into the orbit on July 3, 1997 and on July 4, 1997, it touchdown on its landing site, which is an ancient flood plain full of rockiest parts in northern hemisphere known as Ares Vallis. Pathfinder was the first mission to use a parachute in its descent phase and a globe of airbags to act as a cushion upon the 
surface impact. Upon landing and after airbag deflation the lander weighed $370 \mathrm{~kg}$. The lander includes the rover named Sojourner which was a small rover weighing 15.6 $\mathrm{kg}$ along with the science instruments mounted on it. The six-wheel, rocker-bogie suspension system provides more excellent stability and obstacle crossing capability to the rover on uneven Martian surface. The rover carried three cameras and a set of experiments for testing material adherence and wheel abrasion. A laser system, along with the cameras helped to detect and avoid the obstacles. Both the lander and the rover carried scientific observations and provided engineering data on the new technologies which include Imager for Mars Pathfinder (IMP), Atmospheric Structure Instrument and Meteorology Package (ASI/MET) and Alpha Proton X-ray Spectrometer (APXS). The IMP was used to carry out several atmospheric investigations. The camera also used to measure Aerosol opacity, water vapor abundance and characterize dust particles. The ASI/MET acquired atmospheric information during the descent of the lander through the Martian atmosphere and during the entire landed mission. The APXS was designed to determine the elements that make up the soil and rocks on Mars. The chemical analysis performed by Pathfinder during its mission shows that some rocks contain a high amount of silica. The rounded pebbles and cobbles on the ground and sockets and pebbles in some rocks suggest the formation of conglomerates during the presence of water in the past. The precise measurement of the lander's location and rotation of the poles is obtained by radio tracking the Pathfinder. Upon investigation of characteristics, it is seen that the airborne dust in the atmosphere is full of Maghemite, which is a magnetic form of iron oxide. Whirlwinds called dust devils were also imaged and measured with the pressure, temperature and wind sensors. The formation of clouds containing water ice is also seen in the early time of the day. Abrupt fluctuations in temperature were noted in the atmosphere during the daytime. Due to the presence of a little amount of dust in the atmosphere, the yellowish-brown color is also noted. During its mission, Pathfinder achieved all its goal by collecting 16,500 pictures of Mars from its lander and 550 pictures from its rover. The Pathfinder ended its mission on September 27, 1997 (Fig. 7) (Golombek et al., 1999).

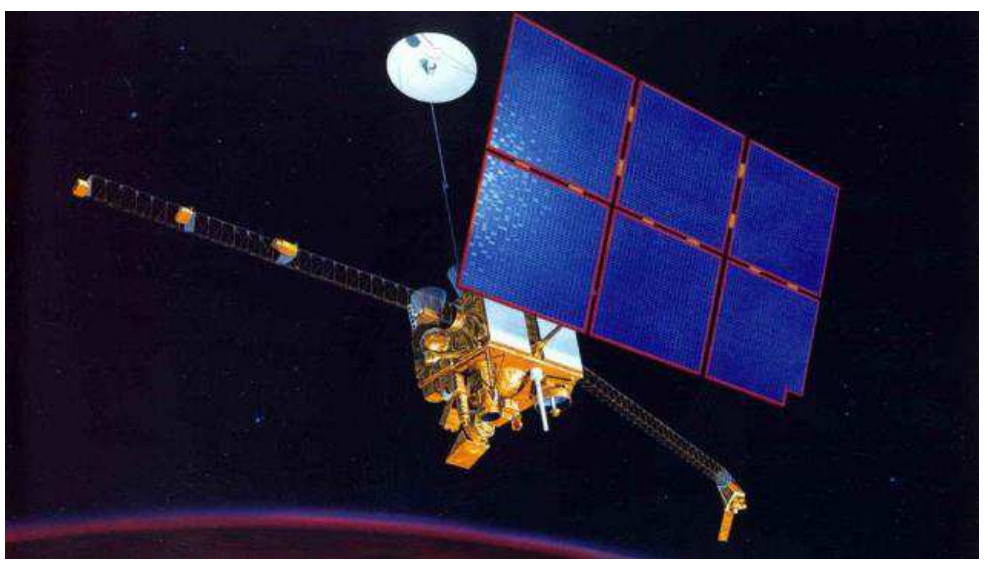

Fig. 5: Mars Observer Spacecraft

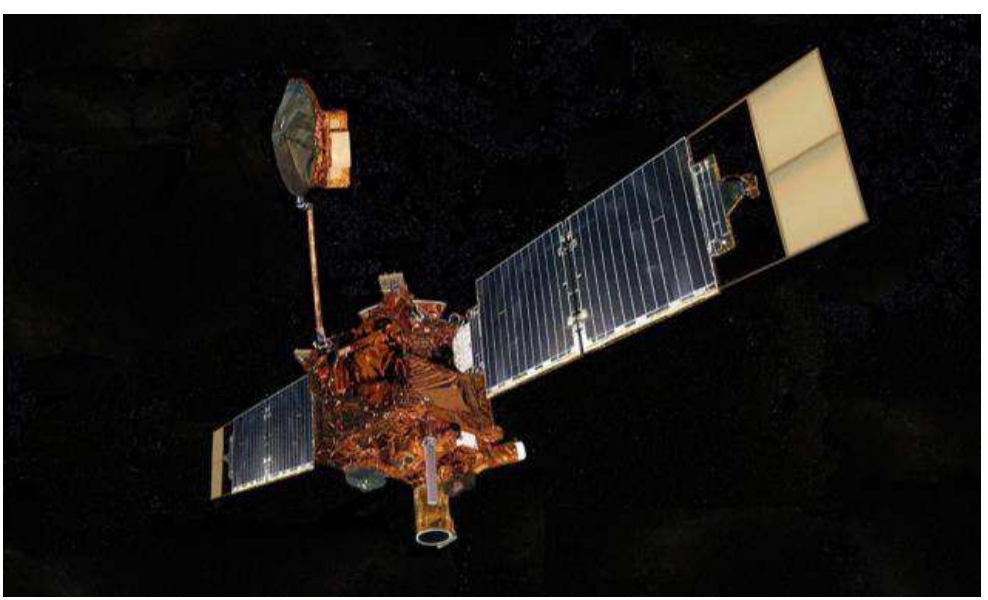

Fig. 6: Mars Global Surveyor Spacecraft 


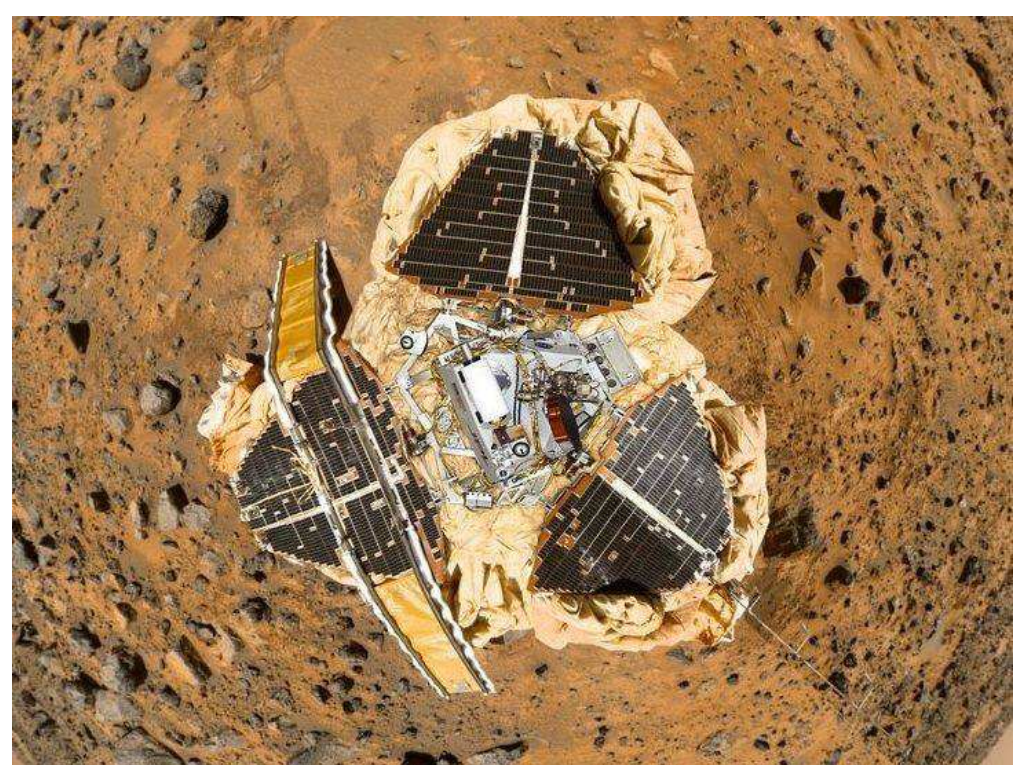

Fig. 7: Mars Pathfinder Spacecraft

\section{Mars Climate Orbiter}

NASA's Mars Climate Orbiter was the first Martian weather satellite to study and monitor the Martian climate and atmosphere. The spacecraft was also designed to act as a communication relay for the next lander missions. The launch took place on December 11, 1998, on two-stage Delta-II vehicle. The two scientific apparatus were mounted on spacecraft, Pressure Modulator Infrared Radiometer (PMIRR) and Mars Color Imager (MARCI). The radiometer was designed to understand and construct the vertical profile of the planet's atmosphere. The MARCI consists of two cameras for capturing the pictures of the Martian atmosphere and surface using wide-medium angles. Due to the conversion errors in units, on September 23, 1999, NASA concluded that the spacecraft entered too low into the Mars orbit and possibly burned up completely (Fig. 8) (Stephenson et al., 1999).

\section{Mars Polar Lander/Deep Space 2}

NASA's Mars Polar Lander also called Mars Surveyor '98 Lander, was designed under the New Millennium Program (NMP). The launch of spacecraft took place on January 3, 1999, on a Delta-II rocket. The spacecraft was designed to touch down at a unique region of Mars close to the southern pole to study the soil and climate of the red planet. The three scientific instruments which include Mars Descent Imager (MARDI), Light Detection and Ranging (LIDAR) and Mars Volatiles and Climate Surveyor (MVACS) were mounted on the lander. The lander also consists of two small impactor probes named as Scott and Amundsen. The two microprobes were designed to penetrate the
Mars surface up to 1 meter to find clues of the presence of water ice and test ten new technologies. Unfortunately, upon arrival in the Martian atmosphere contact with the spacecraft was lost on December 3, 1999 and the mission was ended (Fig. 9) (JPL, 2000).

\section{Mars Phoenix}

The Phoenix Mars Mission was the first mission in NASA's Mars Scout Program (MSP). The launch of spacecraft took place on a Delta-II rocket on August 4, 2007. The Phoenix mission was designed to study the history of water and search for complex organic molecules in the ice-rich soil of the Martian arctic while monitoring polar climate. Phoenix spacecraft used a lander and also carried a sophisticated suite of instruments. After its journey, Phoenix entered the Mars atmosphere on May 25, 2008 and touched down on the far side of north on Green Valley of Vastitas Borealis. The robotic arm on Phoenix dig the surface soil layers to rich the water ice layer and bring samples to lander for further analysis. One of the instrument checked for the presence of water and carbon compounds by heating soil and studying the vapors. While other instrument checked and analyzed the dissolution products by adding water into soil samples. The mission was launched to achieve the three science objects which were designed to study the history of water in all the forms, to study the arctic soil to investigate the potentiality of the planet to sustain life and to examine weather in Polar Regions. The lander was also equipped with the lot of scientific instruments which includes Surface Stereoscopic Imager (SSI), Robotic Arm Camera (RAC), Thermal and Evolved-Gas Analyzer, the Meteorological Station (MET), Mars Descent Imager (MARDI) and the Microscopy, Electrochemistry and Conductivity 
Analyzer (MECA). The camera mounted at the end of the robotic arm provided close-up images of the ice and soil layers. The optical and atomic force microscopes examined samples of mineral grains. Four electrochemical cells measured the number of dissolved salts and the level of alkalinity. The SSI captured clear pictures of the landing site and surroundings as well. The meteorological station monitored any changes in water abundance, dust, temperature and other variables in the atmosphere. After three months, the mission ended on November 2, 2008 (Fig. 10) (Amos, 2008).

\section{Spirit and Opportunity}

The Spirit and Opportunity were NASA's two robotic Mars geologist. Spirit and Opportunity were designed under the Mars Exploration Rover Project to seek evidence of potentiality of the red planet of supporting life. The twin robots, Spirit and Opportunity, were lofted from Florida's Cape Canaveral Air Station on three stages Delta-II rocket. The launch of Spirit took place on June 10, 2003, from Launch Complex 17A and Opportunity was launched on July 8, 2003, from Launch Complex 17B, respectively. With far greater mobility, the twin rovers of Mars Exploration Rover (MER) project continued their pursuit of geological clues to find the habitability of the planet. One of the toughest jobs was to find the landing site that would offer evidence of past liquid water along with the safe landing. After the two years of intensive study, 155 potential landing sites have been suggested out of which only two sites which were on the opposite sides of the red planet have been shortlisted for both the robotic explorers. On January 4, 2004, Spirit entered into the Mars orbit and touched down at impact crater of about $150 \mathrm{~km}$ in diameter named as Gusev Crater. While Opportunity started orbit insertion on January 25, 2004 and landed near the Martian equator at Meridiani Plannum. After the successful touch down these two rovers started their mission to seek any evidence of the habitability of the planet in the past by determining the ancient climate and water at different sites. The mission was designed to accomplish the science objectives which were to hunt and examine diversity of rocks and soils to find any sign of water activity in the past, to perform an investigation of landing sites that holds the probability of chemical and physical signs due to the action of past water, to determine the nature of geological processes from surface morphology and chemistry, perform calibration and validation of surface observations to determine the accuracy and effectiveness of the instruments mounted on Mars Reconnaissance Orbiter (MRO), search for ironbearing carbonates which were formed due to the presence of water, determine the geological operations that have created the soil and rocks on the planet, perform an investigation of the environmental conditions at the time water was present and searched for any geological clues for potentiality of the planet. Both the rovers carried a package of scientific instruments which were known as Athena science payload.

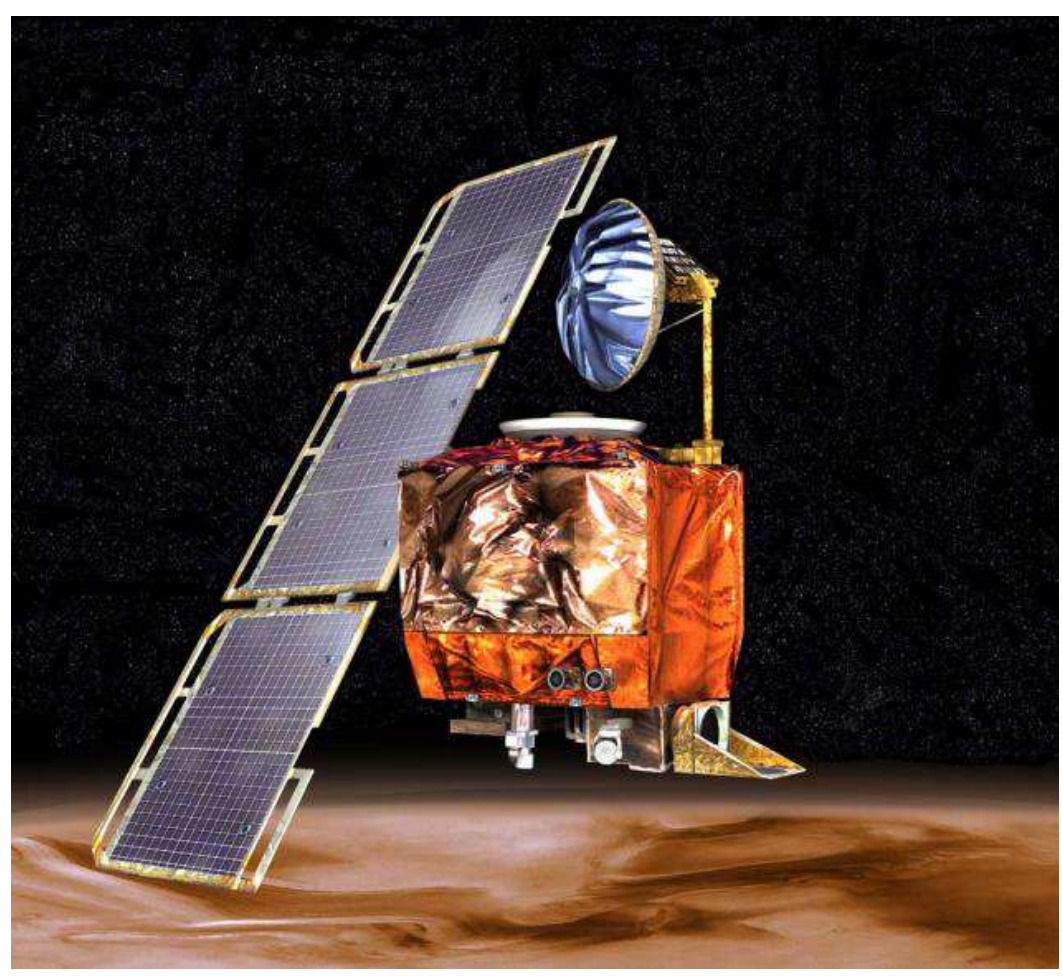

Fig. 8: Mars Climate Orbiter Spacecraft 


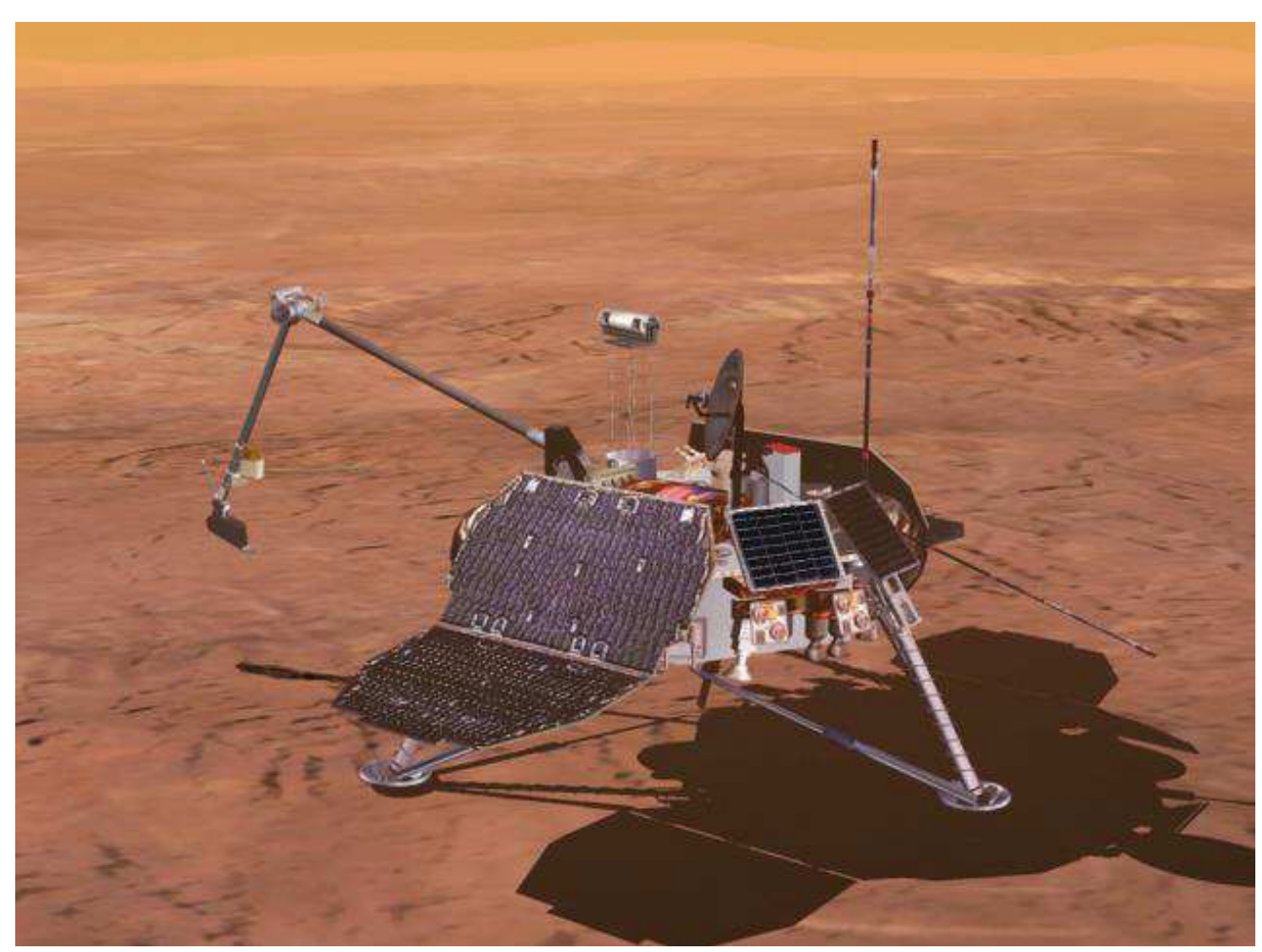

Fig. 9: Mars Polar Lander

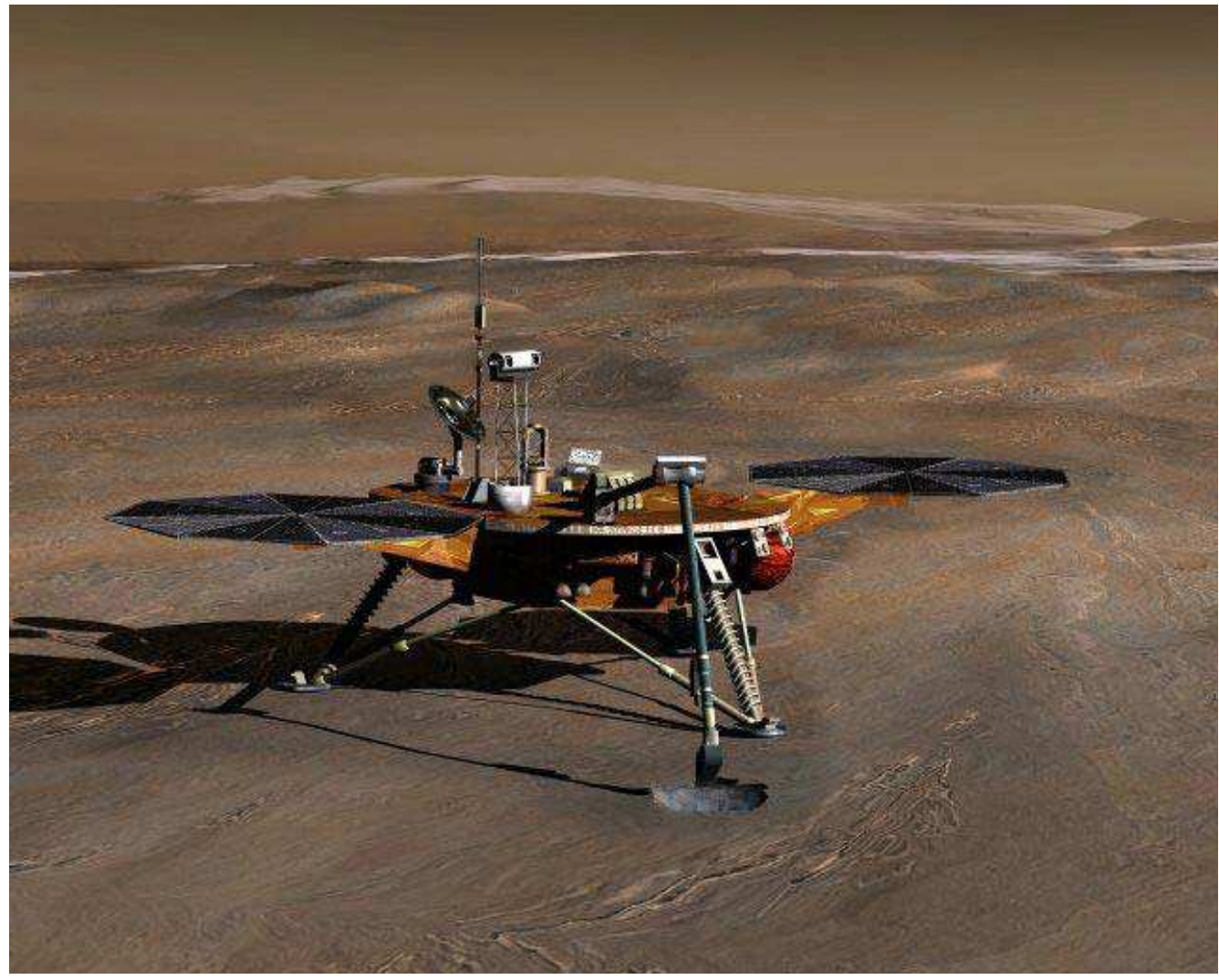

Fig. 10: Mars Phoenix Lander 
The package includes Panoramic Camera, Mini-Thermal Emission Spectrometer, Microscopic Imager, Mossbauer Spectrometer, Alpha Particle X-Ray Spectrometer, Rock Abrasion Tool and Magnet Arrays. Panoramic Cameras consisting of two high-resolution color stereo cameras captured panoramas of the Martian surface with unprecedented detail along with complementing the rover's two navigation cameras. The panoramas provided information on surface features of landing sites along with the distribution and shape of rocks. The MiniThermal Emission Spectrometer helped scientists determine the composition of minerals and to make the selection of specific rocks and soil to analyze in detail by capturing the infrared radiation emitted by objects. The Microscopic Imager provides extreme close-up images of rocks and soil to interpret data about minerals and elements. The Mossbauer Spectrometer determined the amount and composition of iron-containing minerals with high accuracy. The Alpha Particle X-Ray Spectrometer helped to determine the elements that makeup rocks and soils accurately. The Rock Abrasion Tool helped in exposing the fresh surface of the rock by grinding the layer above it. The Magnet Arrays acted as a collecting tool for airborne dust to analyze by science instruments. Both the robotic vehicles have captured and sent thousands of high-resolution color images as well as panoramas of the planet. The rovers collected and transmitted an enormous amount of data capable enough for scientists to reconstruct the past of the planet when it holds the water. The possibility of the planet to sustain life in the past has been revealed from the data of the rovers. Spirit's discoveries revealed that the formation of the planet in the past was due to the volcanic activities, the presence of subsurface liquid water and impacts. While Opportunity found the evidence of habitability of the planet due to the presence of micro salt watered body in the samples of rocks. Also, the rover revealed the presence of sulfate-rich rocks and sands formed due to the evaporation of inter-dune playa lakes after exploring the Endurance and Eagle craters. Though the designed life of three months was achieved by both the vehicles in April 2004, the excellent health helped them to continue their journey to search for the presence of extinct life on Mars. After exploring the planet well beyond its lifetime Spirit ended its mission on March 22, 2010. Whereas, Opportunity made the record for being the first off-Earth robotic vehicle to travel the longest distance of $42 \mathrm{~km}$ on another planet. Finally, on February 13, 2019, due to loss of contact, NASA ended the Opportunity's mission (Fig. 11) (Squyres, 2006) (Arvidson, 2006).

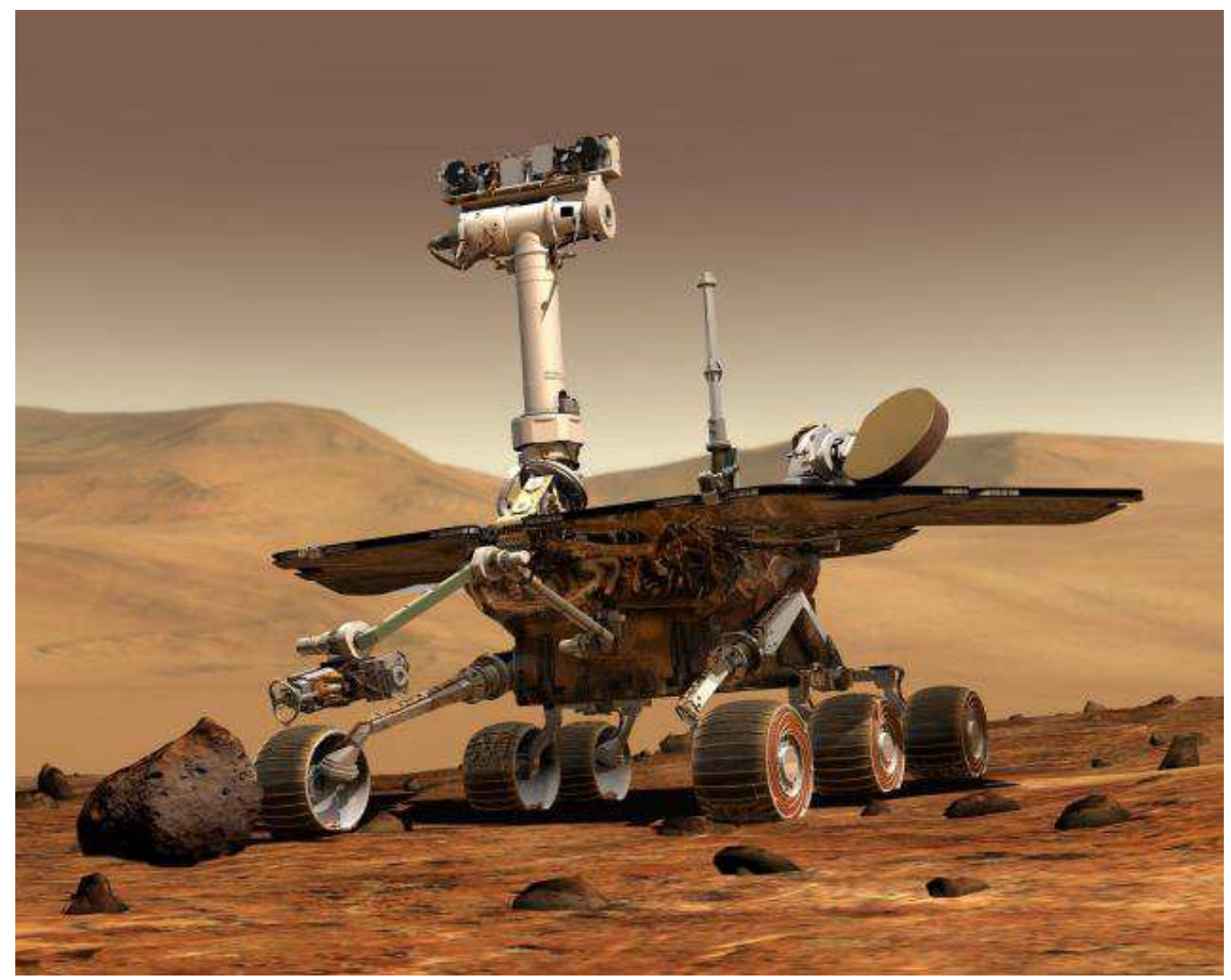

Fig. 11: Spirit and Opportunity Rovers 


\section{Present Missions to Mars}

\section{Mars Express}

NASA has participated in the Mars Express mission, which is the first space exploration mission to Mars developed and built by European Space Agency (ESA). It is one of the rapidly developed spacecraft for the Mars mission. Mars Express consists of an orbiter and a lander. The mission is dedicated to studying the overall atmosphere and environment of the red planet from a quasi-polar orbit. Objectives of the mission are to find any traces of subsurface water and deploy a lander named Beagle-2 on the Martian surface. The launch of the spacecraft took place on June 2, 2003, on Soyuz-FG vehicle. Traveling through the quickest path in space, the spacecraft reached into the Mars orbit on December 25,2003 . The spacecraft carries seven payloads which are High Resolution Stereo Camera (HRSC), Energetic Neutron Atoms Analyser (ASPERA), Planetary Fourier Spectrometer (PFS), Visible and Infrared Mineralogical Mapping Spectrometer (OMEGA), Sub-Surface Sounding Radar Altimeter (MARSIS), The Radio Science Experiment (MaRS) and Ultraviolet and Infrared Mars Atmospheric Spectrometer (SPICAM). The Beagle-2 was designed to detect extinct or extant life on the red planet. However, the lander was unsuccessful to touchdown on the planet's surface. NASA, in collaboration with Italian Space Agency, developed the MARSIS for the spacecraft which have provided the data about the underground water ice, impact craters and layered deposits present under the surface of Mars. Throughout its operations, the spacecraft acts as a communication relay between NASA's various spacecraft and landers. Also, NASA provided the backup tracking system for the mission's critical phases through the Deep Space Network (Fig. 12) (Salese et al., 2019).

\section{Mars Reconnaissance Orbiter}

The Mars Reconnaissance Orbiter (MRO), NASA's first ever mission to conduct an investigation and explore the planet from low orbit blasted off on August 12, 2005, on Atlas V two-stage rocket. The mission is designed to identify and locate the potential landing sites for future crewed missions, investigate the processes that shaped and formed Mars through detailed observations and to act as a communication relay for other missions. The orbiter gets inserted into the Martian atmosphere on March 10, 2006. The spacecraft carries six science instruments which include High-Resolution Imaging Science Experiment (HiRISE), Context Camera (CTX), Mars Color Imager (MARCI), Compact Reconnaissance Imaging Spectrometer for Mars (CRISM), Mars Climate Sounder (MCS), Shallow Surface Radar (SHARAD) and engineering instruments. HiRISE is a most powerful telescopic camera ever sent on an interplanetary mission is designed to provide the photographs of the selected regions on Mars with extraordinary clarity. It will help to plot the 3D data of Mars structure for future space missions. The CRISM will provide the spectral images that are helpful to identify the mineral composition of the Mars surface. The CTX will capture the photographs of swaths, which is $30 \mathrm{~km}$ wide. The MARCI helps to identify any changes in the weather of Mars by plotting the daily global views. MCS will study the water vapor, ice, dust and temperatures in the Mars atmosphere. The SHARAD will map and search for the presence of layers of ice, rock and liquid water present beneath the surface (Fig. 13) (Zurek and Smrekar, 2007).

\section{Mars Science Laboratory (MSL)}

The Mars Science Laboratory (MSL) mission is designed under the Mars Exploration Program (MEP) to search for clues of the potentiality of Mars to sustain past and present microbial life. Also, the mission is designed to determine the presence of water and understand climate and geological processes, which will be helpful for future human missions. The launch of MSL consisting of a rover Curiosity took place on November 26, 2011, on Atlas V rocket. After the voyage of nine months, on August 6, 2012, rover Curiosity touched down on the surface of Mars at Gale Crater. The technology used to land the rover on the red planet is entirely new. During descend the spacecraft ejected the parachute and before touch down the spacecraft fired the rockets to hover and a tether to land the rover on the surface of Mars. After the rover touched successfully, the landing system flew to a safe distance after cutting the tether. The multi-mission radioisotope thermoelectric generator powers the rover weighing $899 \mathrm{~kg}$. Curiosity is designed to easily cross the formidable obstacles and travel on the planet with the speed of $30 \mathrm{~m}$ per hour. The rover carries a total of ten scientific instruments weighing $75 \mathrm{~kg}$. Along with these instruments rover also includes seventeen cameras such as two Mast Cameras (MastCam), one Chemistry and Camera Complex (ChemCam), four Navigation Cameras (navcams) and eight Hazard Avoidance Cameras (HazCams). The Alpha Particle X-ray Spectrometer (APXS) will help to determine the elemental composition of the samples irradiated with alpha rays by mapping spectra of X-rays re-emitted by them. CheCam is made up of LaserInduced Breakdown Spectroscopy (LIBS) and Remote Micro-Imager (RMI) telescope to analyze and identify the mineral composition present in rocks and soils through laser targeting. Sample Analysis at Mars (SAM) will analyze the chemical state of the elements essential for life and also examine the gases from the Martian atmosphere. The Mars Hand Lens Imager (MAHLI) will capture the microscopic images of the soils and rocks. MastCam will provide the color images of the Martian 
terrain with Medium Angle Camera (MAC) and NarrowAngle Camera (NAC). Rover Environmental Monitoring Station (REMS) will measure the regular and periodic changes in the planet's weather. The Radiation Assessment Detector (RAD) tool will detect the high energy atomic and subatomic particles reaching on the surface of Mars from the Sun. The Dynamic Albedo of Neutrons (DAN) will help to detect the presence of water at or near the planet's surface. The Mars Descent Imager (MARDI) will capture the full-color video of the surface while performing touchdown operation. During its operation, Curiosity found chemical and mineral clues of the potentiality of Mars to sustain life. Though the mission lifetime for the Curiosity was of one Martian year, the rover is still exploring the planet for the clues of habitability (Fig. 14) (Grotzinger et al., 2012).

\section{Mars Atmospheric and Volatile EvolutioN (MAVEN)}

The MAVEN is the first spacecraft launched by NASA to explore the higher atmosphere of the red planet and the second mission developed under Mars Scout Program. The mission is designed to understand the planet's potentiality and changing climate over time by studying how the atmosphere and water of the red planet are lost. MAVEN is launched on November 18, 2013, on Atlas V-401 rocket and get successfully inserted into an elliptical orbit around Mars on September 22, 2014. The primary objectives of the mission are to understand the processes controlling the planet's atmosphere along with its composition, measure the rate at which gases are lost into space and processes controlling them. To understand whole planet's atmosphere the spacecraft consists of eight scientific instruments which includes Solar Energetic Particle (SEP), Solar Wind Ion Analyzer (SWIA), Solar Wind Electron Analyzer (SWEA), Magnetometer (MAG), Super Thermal and Thermal Ion Composition (STATIC), Imaging Ultraviolet Spectrograph (IUVS), Neutral Gas and Ion Mass Spectrometer (NGIMS) and Langmuir Probe and Waves (LPW). SEP will determine the effect of particles coming from the Sun on the planet's atmosphere. SWIA will measure the amount of ion entering the planet's atmosphere. STATIC will help to measure the composition and velocity of high energy ions in Mar's upper atmosphere. LPW will determine the ionosphere boundary and density along with the temperature of ionospheric electrons. SWEA will measure the energy and angular distribution of solar wind in Mar's atmosphere. MAG will collect the data about the magnetic environment as it travels through the ionosphere. NGIMS will help to measure the number of neutral ions and their composition in the planet's atmosphere. IUVS will measure the chemical makeup of ionosphere and upper atmosphere of the planet (Fig. 15) (NASA, 2008).

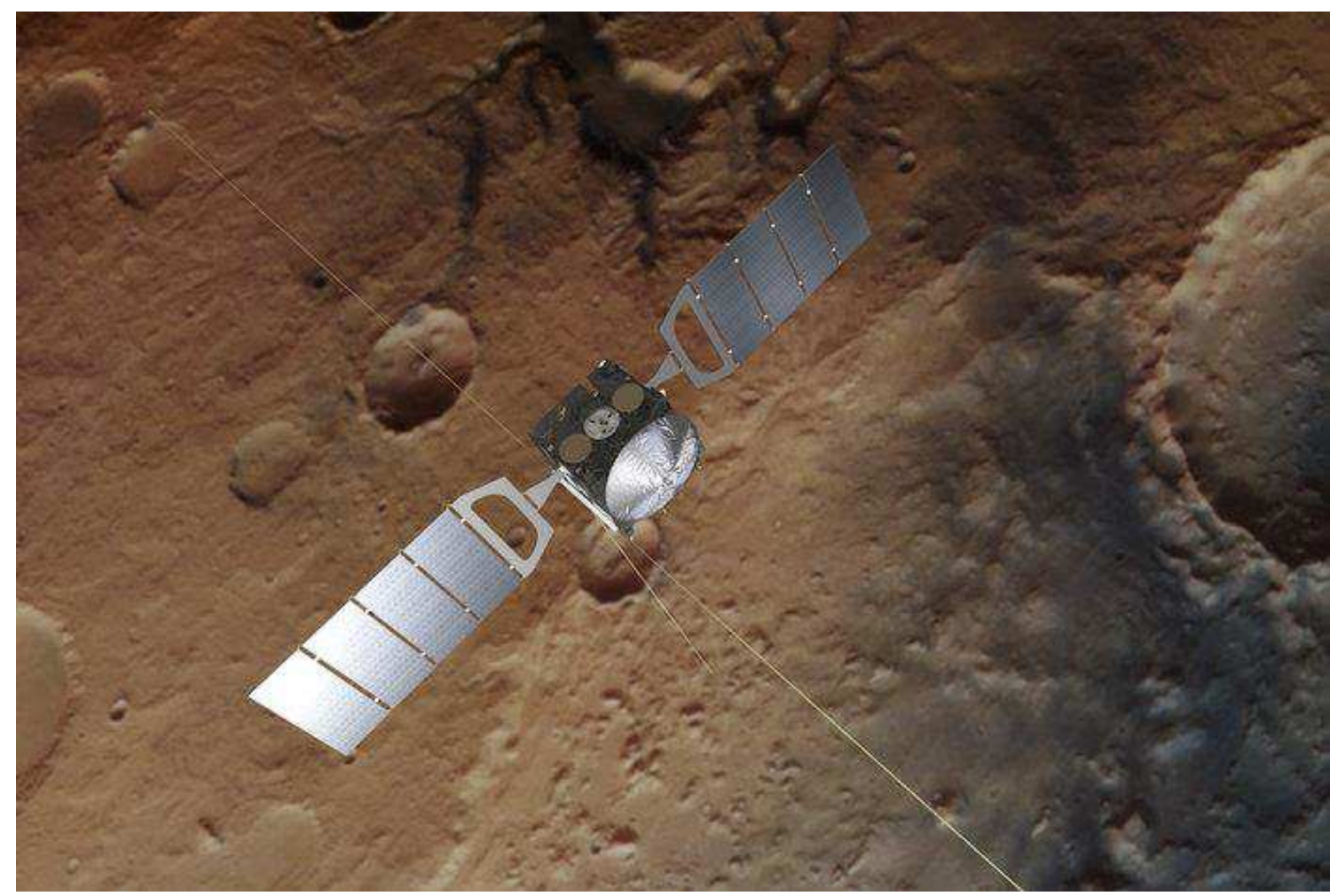

Fig. 12: ESA's Mars Express Orbiter 


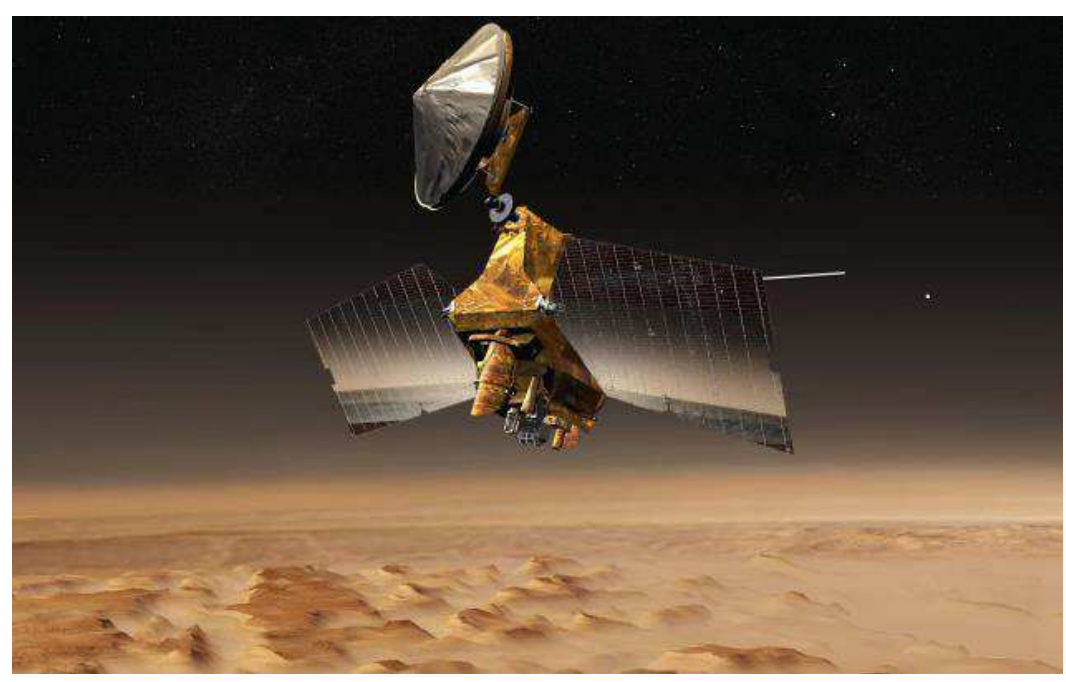

Fig. 13: Mars Reconnaissance Orbiter

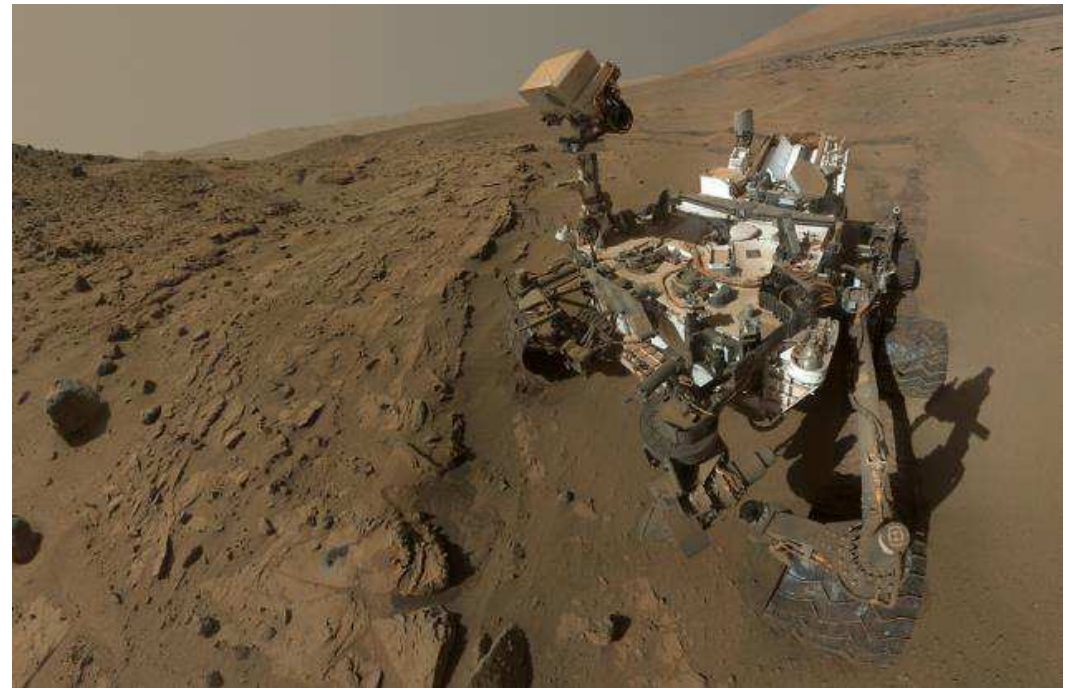

Fig. 14: Mars Science Laboratory Curiosity Rover

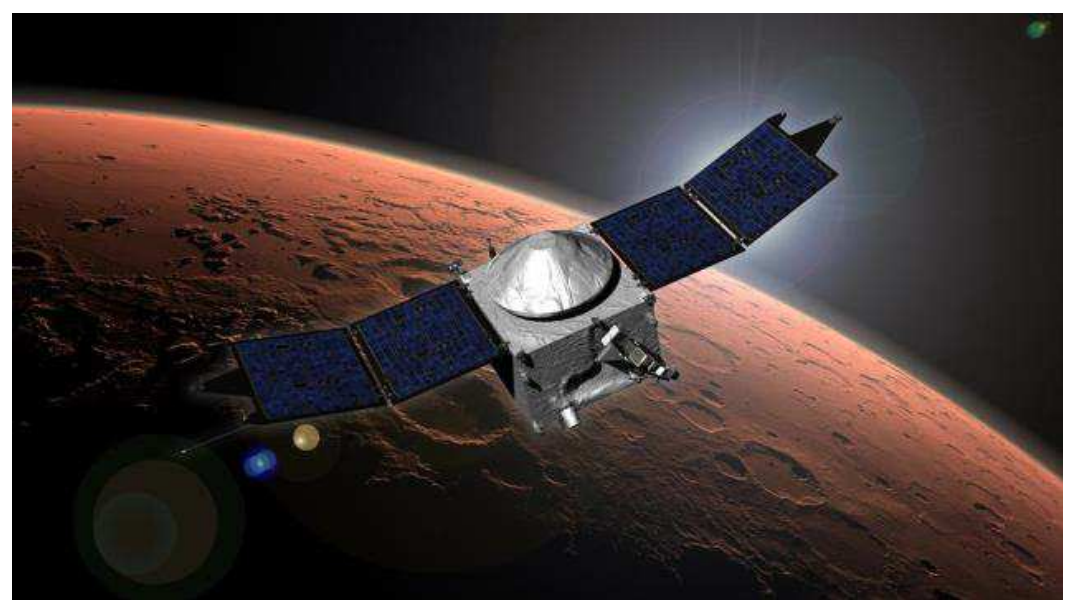

Fig. 15: Mars Atmosphere and Volatile EvolutioN (MAVEN) 


\section{ESA's ExoMars Orbiter}

The ExoMars Program, known as Exobiology on Mars, consists of two mission designed to investigate the presence of past life on Mars. The Trace Gas Orbiter (TGO) is the first mission under the ExoMars Program designed to seek any sign of the existence of Methane and other inert atmospheric gases on the planet which could be signatures of active geological and biological activities. The ExoMars Program is a joint program between the European Space Agency (ESA) and the Russian Space Agency (Roscosmos). The launch of spacecraft took place on March 14, 2016, on Proton-M vehicle from Baikonur Cosmodrome, Russia. The spacecraft also consists of Schiaparelli, an Entry, descent and landing Demonstrator Module (EDM). On October 19, 2016, the EDL module got inserted into the Mars orbit. Unfortunately, the contact with the module lost just before the touchdown. The NASA contributed to this program by providing the telecommunication radios known as Electra. The Electra, Ultra High-Frequency radio will act as a communication relay and navigation aid for the rovers, landers present on the Mars surface and the orbiters. It also provides the precise location of landers and rovers operating on the planet's surface. The radio will help to accurately determine the velocity as well as the position of the spacecraft arriving towards the planet with similar radio. The Electra will also provide sufficient coverage for the rovers and landers with low radio power to communicate and send data to the Earth station (Fig. 16) (NASA, 2016).

\section{InSight}

Interior Exploration using Seismic Investigations, Geodesy and Heat Transport (InSight) mission is designed under NASA's Discovery Program to understand the interior of the planet. The launch of InSight took place on May 5, 2018, on two-stage Atlas V-401 rocket. After the journey of six months, on November 26, 2018, the spacecraft inserted into the Mars orbit and touched down at Elysium Planitia. The mission seeks to understand the evolution and formation of Mars by studying processes and its interior structure. The InSight will try to understand the size, thickness and structure of the planet's core, mantle along with its composition and temperature. The mission will figure out the internal seismic activity of the planet and also the frequency of meteorites impact on the planet. To achieve the objectives of the mission, the spacecraft is mounted with $50 \mathrm{~kg}$ of scientific instruments to perform the significant science operations on the red planet. The payload includes Seismic Experiment for Interior Structure (SEIS), Heat Flow and Physical Properties Package (HP3), Rotation and Interior Structure Experiment (RISE), Temperature and Winds for InSight (TWINS) and Laser RetroReflector for InSight (LaRRI) along with the Instrument Deployment Arm (IDA), Instrument Deployment Camera (IDC) and Instrument Context Camera (ICC). SEIS will understand the structure of the planet by measuring the Marsquakes and other internal activities. The HP3 will measure the amount of heat flow through Mars core to better understand the interior of the planet. RISE will precisely measure the rotation of the planet. TWINS will monitor the daily global weather of the planet (Fig. 17) (Ramon et al., 2018).

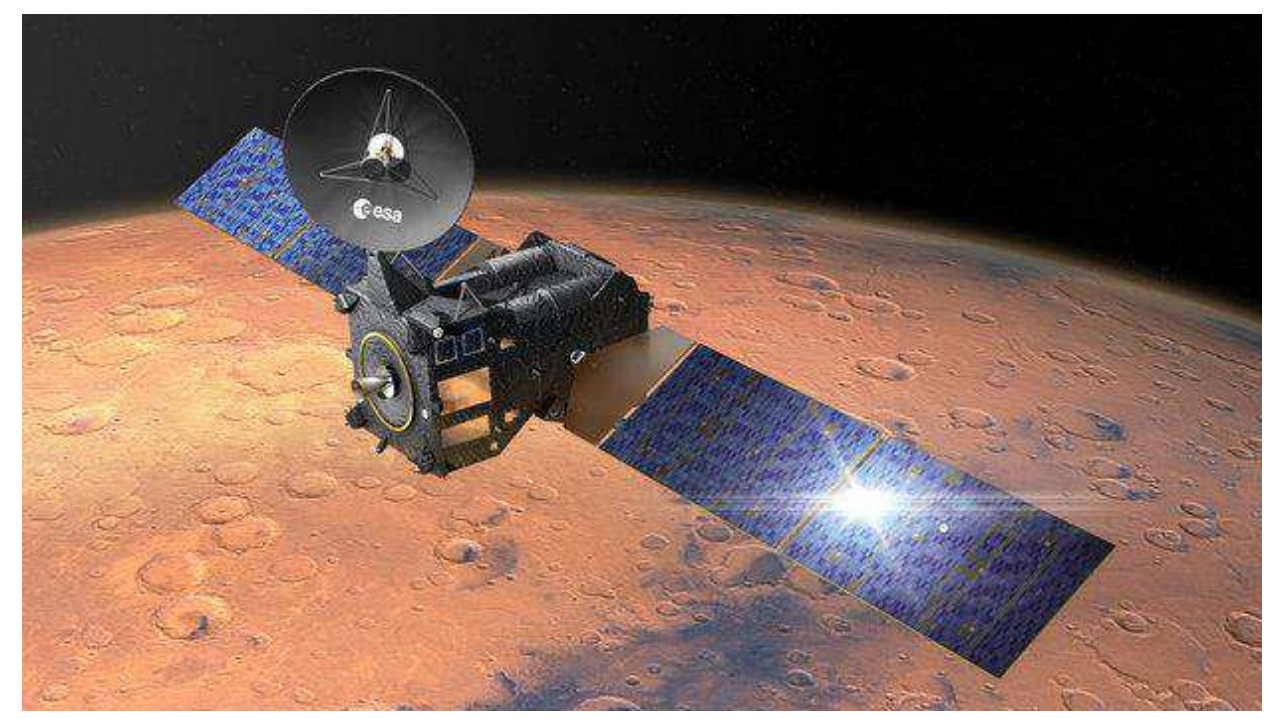

Fig. 16: ESA's Trace Gas Orbiter 


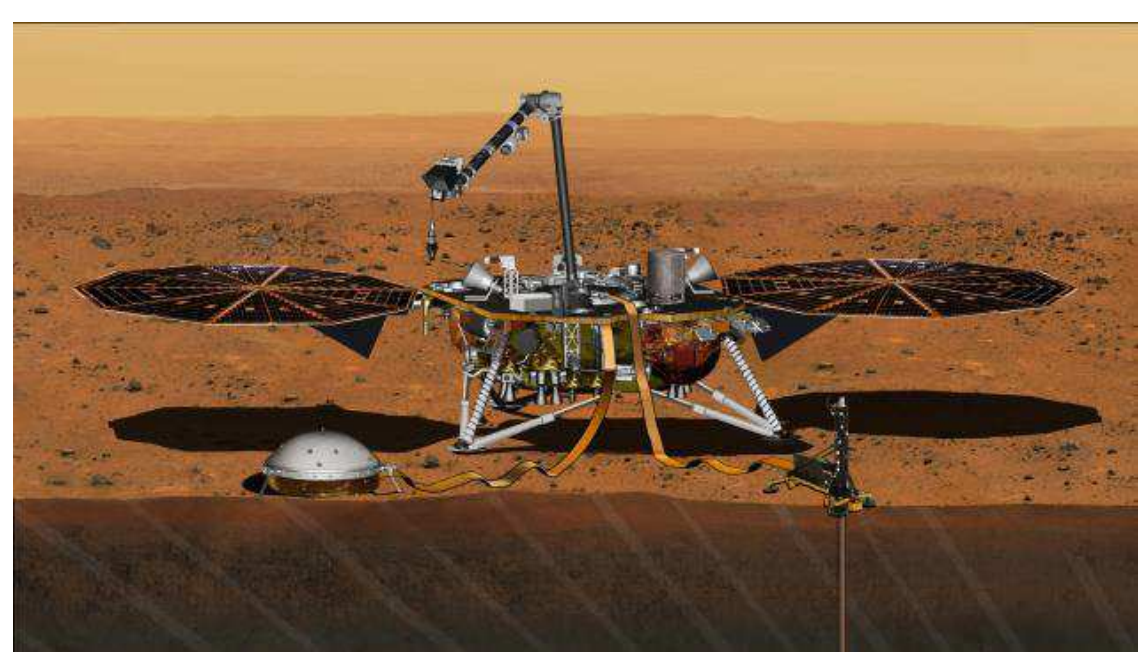

Fig. 17: InSight Lander

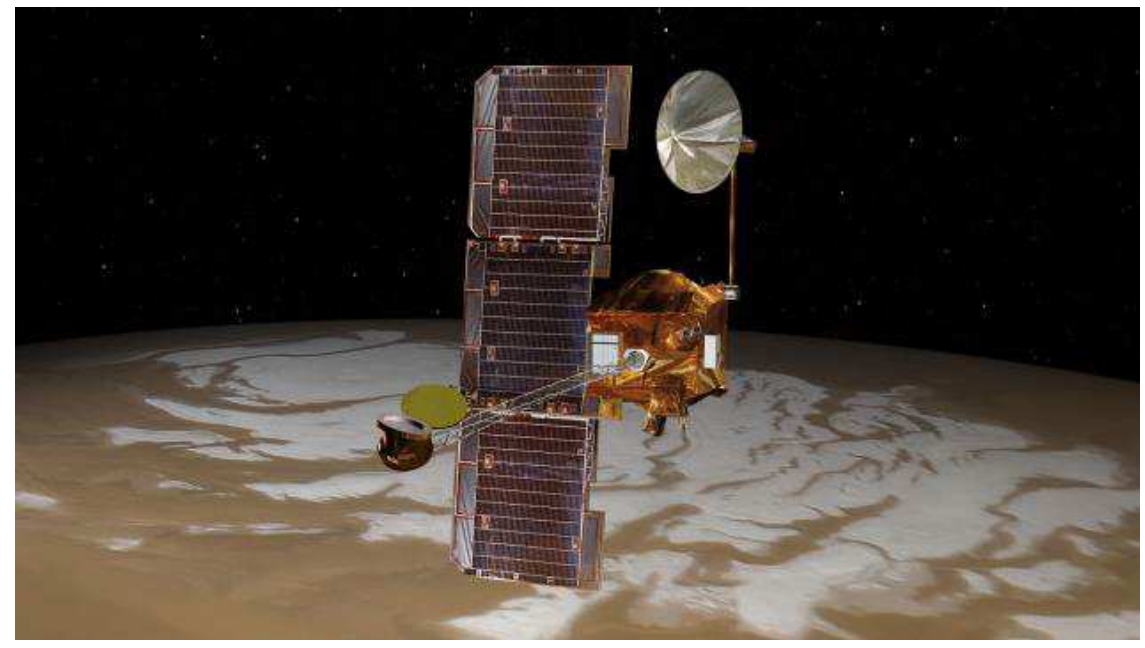

Fig. 18: 2001 Mars Odyssey Orbiter

\section{Mars Odyssey}

Mars Odyssey Mission is one of the missions developed under the Mars Exploration Program to map mineral deposits present in or at Mars and seek any evidence of ancient life and presence of liquid water. The launch took place on April 7, 2001, on Delta II-7925 rocket and after the trip of six months on October 24, 2001 , entered into the Mars orbit. The mission so far plotted the distribution of chemicals and minerals present on Mars along with the mapping of hydrogen buried under the surface in the form of water ice. Also, the spacecraft recorded the data of radiation environment in the low orbit of the Martian atmosphere, which would be helpful for future crewed missions to Mars. The spacecraft carries only three scientific instruments, Mars Radiation Environment Experiment (MARIE), Gamma Ray Spectrometer (GRS) and Thermal Emission Imaging System (THEMIS). THEMIS and GRE will capture the images of the planet in infrared, visible and gamma-ray spectrum in order to detect the minerals and elements on the surface of the planet. MARIE will measure the amount of radiation in the atmosphere using an energetic particle spectrometer. Also, the spacecraft will act as a communication relay for the landers and rovers operating on the planet's surface. Though the objectives of the mission are achieved in two years, the spacecraft is still operational today (Fig. 18) (Christensen et al., 2004).

\section{Future Missions to Mars}

\section{Mars 2020}

Mars 2020, NASA's robotic exploration mission designed under the Mars Exploration Program is scheduled to launch in between July 17, 2020, to August 5, 2020, aboard Atlas V-541 and will touchdown on the Jezero Crater on February 18, 2021. The mission is 
designed to seek any evidence of habitability of the planet in the past and to find biosignatures of earlier microbial life in the rocks and soils at the landing site. Also, to collect individual samples of soils and rocks and set aside on the Martian surface to bring them back on Earth by future sample return missions and conduct an experiment to produce Oxygen from the Martian atmosphere. Also, it will seek any signs of ancient life, presence of liquid water, test improved landing technique and characterize Martian environment for the betterment of future robotic and crewed missions. The scientific instruments mounted on the spacecraft include Planetary Instrument for X-ray Lithochemistry (PIXL), Scanning Habitable Environments with Raman and Luminescence for Organics and Chemicals (SHERLOC), Radar Imager for Mars Subsurface Exploration (RIMFAX), Mars Environmental Dynamics Analyzer
(MEDA), Mars Oxygen ISRU Experiment (MOXIE), SuperCam and Mastcam-Z. Mastcam- $Z$ will capture the high-definition video, panoramic and $3 \mathrm{D}$ pictures of the planet's surface and also assists in rover operations. MEDA will measure and monitor the temperature, pressure, humidity, wind speed and direction and dust size and shape. MOXIE will investigate a technology to produce oxygen utilizing carbon dioxide present in the Mars atmosphere. PIXL will detect the fine scale elemental and chemical composition of minerals present on Mars surface. RIMFAX will provide geologic features of the subsurface of the planet. SHERLOC will detect fine-scale minerals, organic molecules and potential biosignatures in Mars. SuperCam will identify the chemical and mineral composition of rocks and soils, including their molecular and atomic makeup (Fig. 19) (NASA, 2019c).

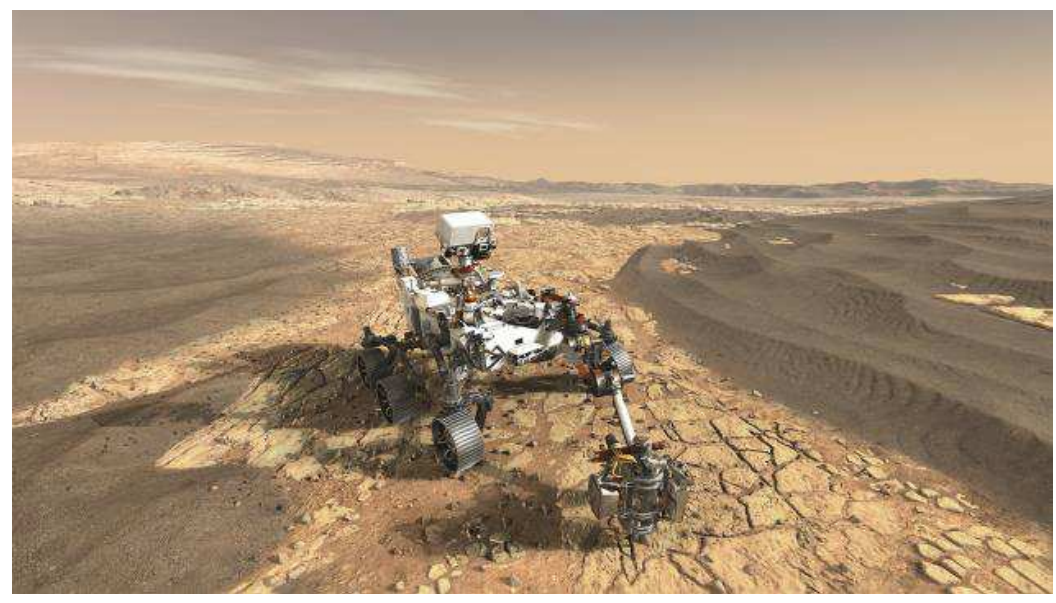

Fig. 19: Mars 2020 Rover

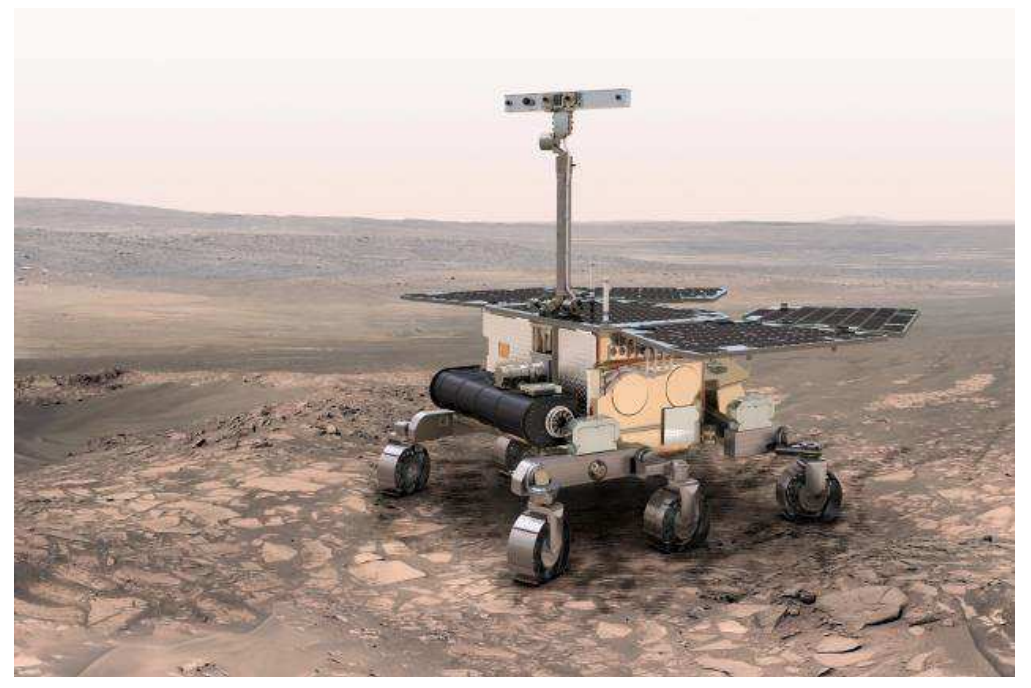

Fig. 20: ExoMars Rover 


\section{ESA's ExoMars Mission 2020}

The ExoMars Mission 2020 is the second mission developed by the European Space Agency (ESA) in cooperation with Russian Space Agency (ROSCOSMOS) under the ExoMars Program. The spacecraft consists of a European rover named Rosalind Franklin and a Russian lander named Kazachok is set to launch in July 2020 and will land on the planet in April 2021. The mission is designed to investigate the local geology and Martian environment and also to seek a sign of earlier and present life on Mars. The lander will consist of seventeen scientific instruments weighing $45 \mathrm{~kg}$ to investigate the surface environment at the landing site. While eleven science instruments weighing $26 \mathrm{~kg}$ mounted on the rover to morphological and chemical signatures of life. NASA's contribution to the mission is in the form of the mass spectrometer and critical electronic components for Mars Organic Molecule Analyzer (MOMA). The MOMA is designed to identify the amount and type of chemicals made up of organic and inorganic compounds present in the Martian rocks and soils (Fig. 20) (Vago, 2017).

\section{Conclusion}

The quest of NASA to better understand the planet led to the launch of several Mars Exploration Missions which includes Orbiting spacecraft's, robotic explorers and scientific landers. Throughout the exploration, these missions have found evidence of potentiality of the planet to sustain microbial life. Also, these missions discovered the presence of water ice buried under the surface and the presence of other natural resources in the Martian environment. The data collected and scientific operations carried out by these missions will be utilized in the future robotic or crewed missions to Mars. The exploration missions to the red planet could be a valuable stepping stone towards crewed missions to Mars. The dream of terraforming and colonizing the red planet will only be achieved by the interaction of future robotic and crewed missions together.

\section{Acknowledgement}

The author would like to thank Prof. Atiksha Sharma for her constant support and encouragement.

\section{Ethics}

The author declare that there is no conflict of interest regarding the publication of this article.

\section{References}

Albee, A., R. Arvidson, F. Palluconi and T. Thorpe, 2001. Overview of the mars global surveyor mission. J. Geophys. Res., 106: 23291-23316.

DOI: 10.1029/2000JE001306
Amos, J., 2008. NASA mars mission declared dead. $\mathrm{BBC}$.

Arvidson, R.E., 2006. Overview of the spirit mars exploration rover mission to gusev crater: Landing site to backstay rock in the columbia hills. J. Geophys. Res., 111: E02S01- E02S01.

DOI: 10.1029/2005JE002499

Christensen, P.R., B.M. Jakosky, H.H. Kieffer, M.C. Malin and J. McSween et al., 2004. The Thermal Emission Imaging System (THEMIS) for the mars 2001 Odyssey Mission. Space Sci. Rev., 110: 85-85. DOI: 10.1023/B:SPAC.0000021008.16305.94

Golombek, M.P., R.C. Anderson, J.R. Barnes, J.F. Bell and N.T. Bridges et al., 1999. Overview of the mars pathfinder mission: Launch through landing, surface operations, data sets and science results. J. Geophys. Res. Planets, 104: 8523-8553.

DOI: 10.1029/98JE02554

Grotzinger, J.P., J. Crisp, A.R. Vasavada, R.C. Anderson and C.J. Baker et al., 2012. Mars science laboratory mission and science investigation. Space Sci. Rev., 170: 5-56. DOI: 10.1007/s11214-012-9892-2

JPL, 1993. Return to the red planet: The mars observer mission. Jet Propulsion Laboratory.

JPL, 2000. Report on the loss of the mars polar lander and deep space 2 missions. Jet Propulsion Laboratory.

Moore, H.J., R.E. Hutton, R.F. Scott, C.R. Spitzer and R.W. Shorthill, 1977. Surface materials of the Viking landing sites. J. Geophys. Res., 82: 4497-4523.

NASA, 1969. Mariner-mars 1969 a preliminary report. NASA, SP-225, Wash., DC.

NASA, 1973. Mariner mars 1971 mars orbiter missions, NASA-JPL. Unnumbered, Pasadena, CA.

NASA, 2008. MAVEN fact sheet. https://www.nasa.gov/sites/default/files/files/MAVE NFactSheet_Final20130610.pdf

NASA, 2016. ExoMars orbiter mission. NASA's Participation in ESA's.

NASA, 2019a. Mars exploration program. https://mars.nasa.gov/\#mars_exploration_program

NASA, 2019b. NASA facts, mariner to mercury, Venus and mars. https://mars.nasa.gov/files/mep/MarsMission-Mariner-Fact-Sheet.pdf

NASA, 2019c. Overview-Mars 2020 Rover. https://mars.nasa.gov/mars2020/mission/overview/

Ramon, P.D.P., B. Banerdt and T.A. Hoffman, 2018. Update status and overview of NASA's insight mars mission. Proceedings of the 69th International Astronautical Congress, Oct. 1-5, Bremen, Germany.

Reiff, G.A., 1966. Mariner IV: Developing the scientific experiment. Sci. New Series, 151: 413-417.

DOI: $10.1126 /$ science. 151.3709 .413 
Salese, F., P. Monica, N. Alicia, S. Gene and O.G. Gabriele, 2019. Geological evidence of planet $\square$ wide groundwater system on mars. J. Geophys. Res. Planets, 124: 1-5. DOI: 10.1029/2018JE005802

Soffen, G.A. and C.W. Snyder, 1976. The first viking mission to mars. Sci. New Series, 193: 759-766. DOI: $10.1126 /$ science. 193.4255 .759

Squyres, S.W., 2006. Two years at meridiani planum: Results from the opportunity rover. Science, 313 : 1403-1407. DOI: 10.1126/science. 1130890

Steinbacher, R.H. and N.R. Haynes, 1973. Mariner 9 mission profile and project history. Icarus, 18: 64-74. DOI: 10.1016/0019-1035(73)90173-5

Stephenson, A.G., L.S. Lia, D.R. Mulville, P.J. Rutledge and F.H. Bauer et al., 1999. Mars climate orbiter mishap investigation board phase I report. NASA.

Vago, J.L., 2017. Habitability on early mars and the search for biosignatures with the exomars rover. Astrobiology, 17: 471-510.

DOI: 10.1089 /ast.2016.1533

Zurek, R.W. and S.E. Smrekar, 2007. An overview of the Mars Reconnaissance Orbiter (MRO) science mission. J. Geophys. Res., 112: E05S01- E05S01. DOI: 10.1029/2006JE002701

\section{Figures Sources}

Fig. 1: $\quad$ Source: https://www.jpl.nasa.gov/missions/web/mariner3_4.jpg Fig. 2: Source: https://www.jpl.nasa.gov/missions/web/mariner6_7.gif

Fig. $\quad 3: \quad$ Source: https://www.jpl.nasa.gov/missions/web/mariner9.jpg Fig. $\quad 4: \quad$ Source: https:/www.nasa.gov/sites/default/files/thumbnails/imag e/483308main_aa_3-9_history_viking_lander_1024.jpg Fig. $\quad$ 5: Source: https://www.jpl.nasa.gov/missions/web/mars_observer.jpg Fig. $\quad$ 6: $\quad-{ } \quad$ Source: https://www.jpl.nasa.gov/missions/web/mgs.jpg

Fig. $\quad 7$ : Source: https://marsmobile.jpl.nasa.gov/imgs/2000/09/PIA02652fi.jpg
Fig.

8:

Source: https://www.jpl.nasa.gov/missions/web/mars_climate_or biter.jpg

Fig.

Source:https://mars.nasa.gov/msp $98 / \mathrm{msss} / \mathrm{mars}$ relay/ds $2 \mathrm{mpl}$ role/mpl shadow.jpg

Fig. 10: Source: https://www.jpl.nasa.gov/missions/web/phoenix.jpg

Fig. 11: Source:

https://www.jpl.nasa.gov/missions/web/mer.jpg

Fig.

12:

Source:https://www.esa.int/var/esa/storage/images/esa multimedia/images/2018/04/mars_express/17447281-1eng-GB/Mars_Express_node_full_image_2.jpg

Fig.

Source:https://mars.nasa.gov/layout/mro/images/MRO P IA05490.jpg

Fig.

Source:https://www.nasa.gov/sites/default/files/thumbnai ls/image/yir2014-curiosity-portrait-crop.jpg

Fig.

Source:https://mars.nasa.gov/system/resources/detail_fil es/6589 maven-mars-sunrise-full.jpg

Fig.

16:

Source:

https://www.esa.int/var/esa/storage/images/esa_multimedi a/images/2014/01/exomars trace gas_orbiter/ $\overline{14257285-}$ 3-eng-

GB/ExoMars_Trace_Gas_Orbiter_node_full_image_2.jpg Fig. Source:https://www.jpl.nasa.gov/images/mars/insight/20 151203/pia19811-16.jpgFig.

Source:https:/www.jpl.nasa.gov/images/odyssey/20161 228/pia04816-16.jpg

Fig.

19:

Source:https://mars.nasa.gov/system/resources/detail_fil es/21383_PIA22111_1600.jpg

Fig. 20: Source:http://exploration.esa.int/science-emedia/img/content/images/2016/ExoMars20168_Rover 295w.jpg 